\title{
FIELD STIMULATED SURFACE DIFFUSION OF LITHIUM ON GERMANIUM (100) AND (111) PLANES
}

\author{
YU. SUCHORSKI \\ Department of Physics, Technical University of Lvov \\ Mira St. 12, 290646, Lvov, Ukraine \\ (Received May 21, 1991; in revised form May 27, 1991)
}

Field stimulated surface diffusion of lithium on atomically clean (100) and (111) germanium single crystal planes has been investigated by means of field emission method. Sealed-off field emission tube with a channel multiplier was used. The surface density of lithium adatoms on the germanium surface was determined applying a standard tungsten (112) crystal plane. The dependences of the surface diffusion activation energy on the applied electric field at different lithium coverages have been obtained. The initial dipole moment of $\mathrm{Li}$ adatoms on the $\mathrm{Ge}(100)$ and (111) planes was then determined. The values $1.3 \pm 0.2$ and $1.0 \pm 0.2$ debye obtained for the $\mathrm{Li}-\mathrm{Ge}$ (100) and $\mathrm{Li}-\mathrm{Ge}$ (111) systems respectively are in good agreement with the ones calculated from the work function measurements. The reverse effect of the field influence on the surface diffusion of lithium on $\mathrm{Ge}(100)$ and (111) planes has been observed as the second lithium layer is filling. Relative contributions of the peculiarities of semiconductor substrate and alkali adsorbate on the properties of adsystems are discussed on the basis of experimental results.

PACS numbers: 79.70. $+q, 68.35 . F x$

\section{Introduction}

The small size of lithium adatoms and resulted high sensitivity to the potential relief and electron structure of substrate stimulate the attempts to apply this adsorbate as a "probe" to investigate peculiarities of alkali interaction on solid surfaces. Such an idea turns to be very effective for the case of metal surfaces. The experiments on the lithium adsorption on the rough W (112) [1] and Mo (112) [2] planes have permitted to discover the presence of indirect adatoms interaction through the electron subsystem of the metal substrate. From the studies of the lithium adlayers structure on the $\operatorname{Re}(\overline{1010)}$ and $\operatorname{Re}(10 \overline{10})$ surface regions, which differ by the detailed atomic structure due to the second layer, the relative values of the contributions from different types of adatoms interaction were estimated [3]. 
In spite of recent important results $[4,5]$ for the case of semiconductor surfaces, the thorough understanding of both adatom-adatom and adatom-substrate interactions, at the same level as for metal substrate, has not been achieved yet.

In order to provide progress in this area and to improve the theory the experimental investigation of adsystem on semiconductor surface, in particular, for very low coverages and "responses" of such systems on the external factors are essentially desirable. The studies of the external electric field influence on the behaviour of the adsorbed particles on the metal surface have shown that such factor can be helpful to understand the adsorption phenomena [6-13].

The direct FIM observations of the single adatom behaviour in the external field [8] are effective in this sense. However, the "invisibility" of the alkalis within FIM, caused by the unfortunate relationship of the image and desorbing fields restricts these studies by the influence of the non uniform external field on the eqilibrium distribution of the adsorbate on the tip [9-11] and field stimulated surface diffusion (FSSD) [12-13].

First attempts to inspect the external field influence on the surface diffusion (SD) of the alkalis (K, $\mathrm{Na}[14,15])$ on germanium (100) and (111) have elucidated perspective features of this kind of experiments for the semiconductor surfaces. Therefore we intend to consider in this paper the field stimulated surface diffusion of the lithium on the Ge (100) and (111) planes.

\section{Experimental}

The experiments were carried out using a sealed-off glass field emission tube equipped with an adsorbate flux calibrating device analogous to that used by us previously $[14,15]$.

This tube and associated electronics which are schematically similar to that used by Todd and Rhodin [16] were designed to allow for the field emission, work function, field desorption and surface diffusion measurements due to channeltron-assisted registration of the emission current from the small region of the single plane of the tip.

A low-resistance germanium crystal tip was chosen as the substrate. Its atomically clean surface was achieved by glow discharge of argon [17] with subsequent vacuum field evaporation [18]. Cleaned in such a way the surface is especially suitable for the case of diffusion measurements, since the field evaporation makes possible to obtain a smooth surface cleaned from steps, kinks and other irregularities which influence essentially the surface diffusion process.

This surface cleaning method well-tested from the metal surface $[16,19]$ allows to achieve in the semiconductor case also a near perfect condition of the surface [20] providing the diffusion investigations [21]. The lithium source, similar to that used in studies [1-3], has been applied. The determining of the surface density of lithium adatoms on the surface under consideration has been provided by the use of adsorbate atoms flux calibrating device based on the insertion of a standard crystal plate into the adsorbate flux. The description of this device and surface density determining procedure has been presented by us previously [14].

In this case the (112) tungsten crystal plane as a standard substrate and 
work function dependence versus lithium surface density from the Ref. [1] as a standard data have been chosen to lithium flux calibration.

The work function changes due to adsorption were determined from the slopes of Fowler-Nordheim plots, which correspond to the chosen region of the germanium single plane. The diffusion of adatoms was controlled by the photoelectric method [12] which was modified by usage of the double flexible glassfiber.

The SD activation energy was calculated from the slopes of Arrhenius plots.

\section{Results}

The main aim of our measurements in this work was to obtain dependences of the surface diffusion activation energy $Q_{\mathrm{d}}$ as a function of applied electric field $F$ at different surface densities $N_{\mathrm{a}}$ of the adsorbate.

Since it is convenient to determine the absolute surface density $N_{\mathrm{a}}$ from the work function dependences on $N_{\mathrm{a}}$, therefore the work function changes $\Delta \Phi$ due to adsorption of lithium on the Ge (100) and Ge (111) planes were measured at first. The curves $\Delta \Phi$ corresponding to the Ge (100) and Ge (111) planes are shown in Fig. 1. The zero potential point for both curves is that of the clean surface. One

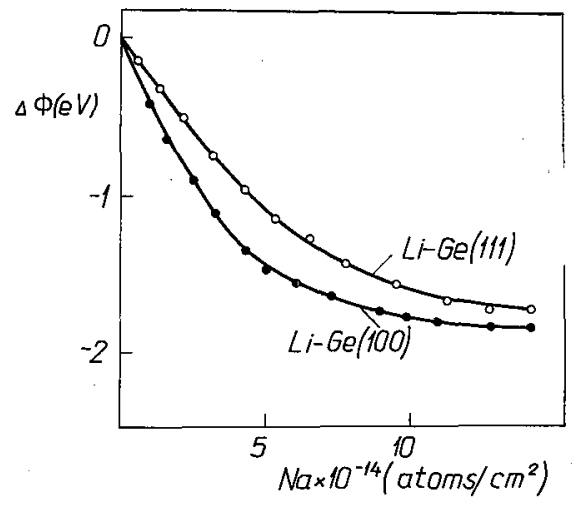

Fig. 1. Work function changes $\Delta \Phi$ of (100) and (111) germanium planes as a function of lithium surface density $N_{\mathrm{a}}$.

has to note that these curves are monotonous and thus recall the corresponding dependences for some rough metal substrates [22]. The initial dipole moments for the "zero coverage" which were calculated conventionally from the initial section of the work function curves (sce Fig. 1) are $1.0 \pm 0.2$ and $0.7 \pm 0.2$, debye for $\mathrm{Li}$ on the Ge (100) and $\mathrm{Li}$ on Ge (111), respectively.

The results of the SD investigations are presented in the Figs. 2 and 3. The set of curves $Q_{\mathrm{d}}(F)$ at various lithium coverages are shown here. The curves obtained are nearly straight lines. Their form is similar to that in the case of electropositive atoms adsorbed on the metal surfaces $[12,13]$ and $\mathrm{K}, \mathrm{Na}$ adsorbed on germanium single planes $[14,15]$. 


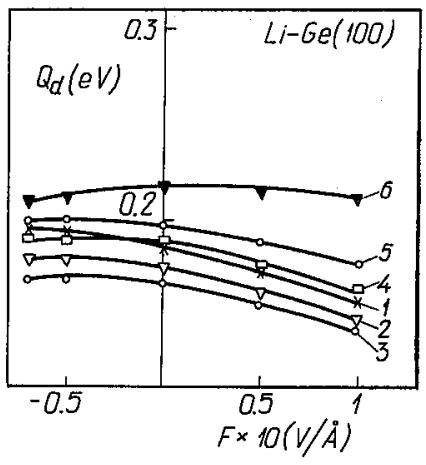

Fig. 2. Surface diffusion activation energy $Q_{\mathrm{d}}$ of lithium adatoms on $\mathrm{Ge}(100)$ as a function of electric field $F$ for different lithium surface densities $N_{\mathrm{a}}: 1.0 .2 \times 10^{14} ; 2.0 .5 \times$ $10^{14}$; 3. $1.0 \times 10^{14} ; 4.1 .5 \times 10^{14} ; 5.2 .0 \times 10^{14} ; 6.7 .0 \times 10^{14}$ atoms $/ \mathrm{cm}^{2}$.

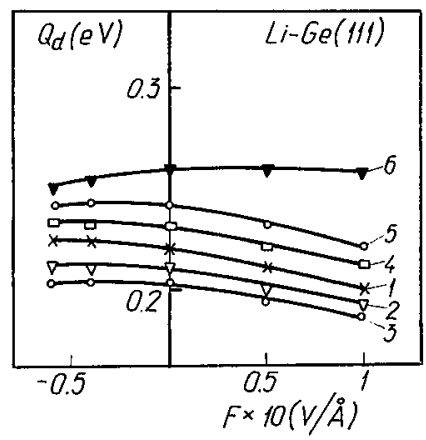

Fig. 3. Surface diffusion activation energy $Q_{\triangleleft}$ of lithium adatoms on Ge (111) as a function of electric field $F$ for different lithium surface densities $N_{\mathrm{a}}: 1.0 .2 \times 10^{14}$; 2. $0.5 \times 10^{14} ; 3.1 .0 \times 10^{14} ; 4.1 .5 \times 10^{14} ; 5.2 .0 \times 10^{14} ; 6.7 .0 \times 10^{14}$ atoms $/ \mathrm{cm}^{2}$.

As the changes of SD energy values due to the external electric field (Figs. 2, 3) can be approximated according to the equation [23]

$$
\left|\Delta Q_{\mathrm{d}}\right|=p_{\mathrm{eff}} \times \boldsymbol{F}+\frac{1}{2} \alpha_{\mathrm{eff}} \times F^{2}
$$

where $p_{\text {eft }}$ is the effective dipole moment of the adatom and $\alpha_{\text {eff }}$ is the effective polarizability, then $p_{\text {eff }}$ and $\alpha_{\text {eff }}$ values can be calculated. The corresponding values of the $p_{\text {eff }}$ as a function of the lithium surface density $N_{\mathrm{a}}$ on $\mathrm{Ge}(100)$ and $\mathrm{Ge}$ (111) planes are given in Fig. 4. The extrapolation of the curves to very low coverages provides the initial dipole moment independently of the work function measurements. The values obtained are $1.3 \pm 0.2$ and $1.0 \pm 0.2$ debye for $\mathrm{Li}-\mathrm{Ge}$ (100) and Li-Ge (111) systems, respectively.

The opposite slope of the curves 6 in the Figs. 2 and 3 manifests the reverse of the field effect at lithium surface density of the order $7 \times 10^{14}$ atoms $/ \mathrm{cm}^{2}$ and 


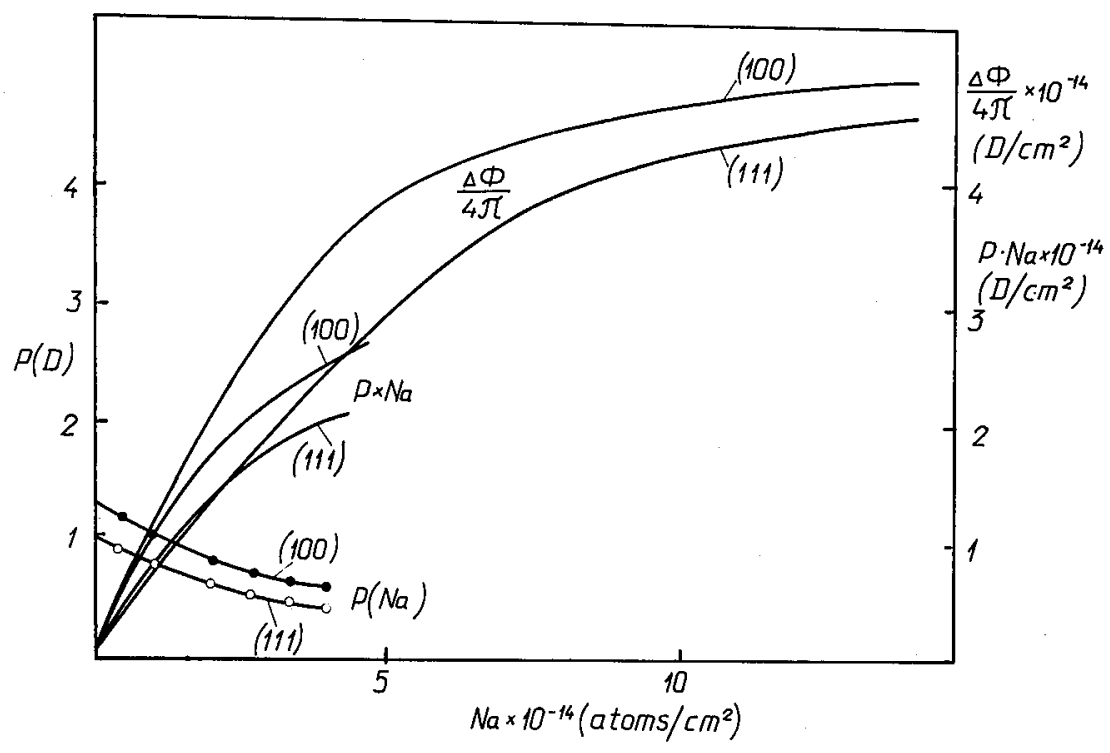

Fig. 4. Dipole moment $p$, electric double layer power $p \times N_{\mathrm{a}}$ and $\Delta \Phi / 4 \pi$ as a function of lithium surface density $N_{\mathrm{a}}$ on Ge (100) and Ge (111) planes.

the slope of these curves leads to the value of the order 0.2 debye for "negative dipole moment". The effective polarizability $\alpha_{\text {eff }}$ of $\mathrm{Li}$ adatoms on $\mathrm{Ge}(100)$ and $\mathrm{Ge}$ (111) planes obtained from the $Q_{\mathrm{d}}(F)$ curves in Figs. 2 and 3 for all investigated coverages does not exceed $5 \AA^{3}$.

\section{Discussion}

In spite of apparent knowledge of the character of binding of alkali adatom with solid surface even for the case of metal substrate the question of its description in terms of ionicity, covalency or metallicity is still actual [24].

In the semiconductor case the actuality of this problem is evident.

The comparison of the dipole moment data independent on the work function measurements with other results, such as for instance the adbond lengths [5], is helpful to solve the question of charge transfer due to alkali adsorption. If one chooses from two aforementioned field-assisted method, the FSSD method is simpler for experimental realization but more complicated for interpretation of the

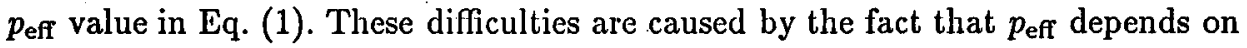
different factors, namely the relationship between the local and average fields and the values of adatom dipole moments in the normal and migration states which differ in general. In accordance with [25], the value of $p_{\text {eff }}$ can be presented as $p_{\text {eff }}=p_{0} k_{0}-p k$, where $p_{0}, p$ are the dipole moments and $k_{0}, k$ are the coefficients connecting the local field with the average surface field in normal and migration state, respectively. Nevertheless, the analysis of previous results both for metal [12, 
13] and semiconductor substrate [14,15] shows that for low adsorbate coverages the values of the dipole moments obtained in such a way are reasonable.

The studies of the case of metal substrate [9-13] have shown that at increasing concentration of adsorbate on the surface the reverse of field action can take place. One can expect similar effect for the semiconductor surface*. We have not obtained this effect in our previous studies $[14,15]$ as they were restricted to the low adsorbate coverages, which possibly were insufficient. In this work the range of coverages investigated was intentionally increased and then the reverse in the field-effect (see curve 6, Figs. 2, 3) has been observed as a result.

As it was noted in [26], it could be hardly possible to compare directly the results of adsorption studies on the metal and semiconductor surfaces. However, as the alkali adsorbate influence prevails in the formation of the adsystem properties for both cases, the comparative analysis in general has sense.

The twofold interpretation of reverse of the field effect has been given: the transition of the electric double layer power through the maximum (the derivative $\mathrm{d}\left(p \times N_{\mathrm{a}}\right) / \mathrm{d} N_{\mathrm{a}}$ influences the value $p_{\text {erf }}$ and even can lead to the change of sign of $\left.p_{\text {eff }}[10]\right)$ or inverse of dipole moment's vector orientation when the second layer is filled [9]. We have a rare opportunity to avoid ambiguous interpretation, because the absence of minimum on the work function curves (Fig. 1) does not necessarily forecast the maximum of the double layer power.

Thus when the second layer is filling $\left(N_{\mathrm{a}}>5 \times 10^{14} \mathrm{~cm}^{-2}\right)$ the lithium adatoms are characterized by a slight, inversely directed dipole moment. We urge to treat this result more as an example of increasing inapplicability of the dipole model at the increasing surface density of adsorbate. This is testified by the discrepancy of the dependence of $p_{\mathrm{efr}} \times N_{\mathrm{a}}$ and $\Delta \Phi / 4 \pi$ (the latter determines the double electric layer power if all the changes of the work function would be caused only by this layer according to well-known formula $\Delta \Phi=4 \pi p \times N_{\mathrm{a}}$ ) on the Fig. 4, as lithium coverage increases. Such a discrepancy and quick decrease of the curves $p\left(N_{\mathrm{a}}\right)$ manifest the drastical lowering of the electron transfer value due to alkali adsorpiion on germanium surface even at low alkali coverages. This result for semiconductor coincides with the new data about low charge transfer to the substrate even for low alkali coverages for the metal surface [24, 27]. For Li this effect is much more pronounced if compared with $\mathrm{K}$ and $\mathrm{Na}$ on $\mathrm{Ge}(100)$ and $\mathrm{Ge}$ (111) planes $[14,15]$. The effective polarizability of $\mathrm{Li}$ adatoms even at low coverages is essentially less than the one for free Li atom $\left(20 \AA^{3}[28]\right)$. This agrees with recent calculations of electron density distribution near the alkali covered semiconductor surface [29]. From these calculations, which consider the Coulomb correlations for the valence electron subsystem of semiconductor, it follows that the external field is screened not only by the adsorbate electrons, but also by those of the substrate.

Our previous papers $[14,15,30]$ contain an analysis of the influence of the atomic relief and the electronic structure of the substrate on some alkali adlayers characteristics on a semiconductor surface. The motivation to consider the case of $\mathrm{Li}$ in this aspect is such that the macroscopic characteristics of $\mathrm{Li}$ adlayers are

*The author is obliged to Prof. A.G. Naumovets who directed author's attention to this obstacle. 
sensitive for the substrate features in addition to the structural adlayer transformations. In spite of similatity of the atomic relief of W (112), Mo (112), Re (1010) planes and the size of the surface cells $(2.74 \times 4.47 \AA, 2.73 \times 4.4 \AA$, and $2.76 \times 4.46 \AA$, respectively [3]) the dependence of the work function due to the lithium adsorption differs considerably [1-3]. This permits to have a hope for elucidation of the influence of substrate properties on the work function and SD results, as the atomic structure influences essentially the mobility of adparticles which follows from the SD studies by the field emission flicker noise method [31,32]. One can expect that at decreasing size of adatom the factor of substrate will become more important as it follows from the comparison of the $\mathrm{K}-\mathrm{Ge}$ and $\mathrm{Na}-\mathrm{Ge}$ systems $[14,15]$.

However, both the work function and SD data presented in this work do not show this effect for lithium, these data for structurally different Ge (100) and Ge (111) planes differ less than it can be expected from the known properties of lithium adlayers on different metal crystal surfaces and known up to date $K$ and $\mathrm{Na}$ adlayers properties on $\mathrm{Ge}(100)$ and $\mathrm{Ge}(111)$ planes $[14,15,30]$.

As it has been shown by Lozovy $[3,33]$ the relative lowering of adatom sensitivity to the peculiarities of substrate may be caused by the prevailing of one of the types of adatom-adatom interaction, namely the advantage of the dipole-dipole interaction ( $\mathrm{K}$ on the $\operatorname{Re}(1010)$, [33]) or the electron exchange interaction ( $\mathrm{Li}$ on the $\operatorname{Re}(1010)$, [3]) each decreasing the sensitivity of both adsorbates relatively to $\mathrm{Na}$, where the two contributions are of similar magnitude [33].

The specific features of the field evaporated germanium surface which is characterized by the high density of surface electron states $\left(\sim 10^{15} \mathrm{~cm}^{-2}\right)$ provide the possibility of the apperance of the long-ranged indirect adatom-adatom interaction through the substrate surface electron band [34]. This type of interaction may prevail in the $\mathrm{Li}$ adlayers on the Ge (100) and Ge (111) field evaporated surfaces and cause the properties observed. A question how to obtain the data on structural transformation in the lithium adlayer on a field evaporated germanium surface, in order to support this supposition, remains open.

\section{References}

[1] V.K. Medvedev, A.G. Naumovets, T.P. Smereka, Surf. Sci. 34, 368 (1973).

[2] M.S. Gupalo, V.K. Medvedev, B.M. Paluch, T.P. Smereka, Fiz. Tverd. Tela 21, 973 (1979).

[3] Ya.B. Lozovy, V.K. Medvedev, T.P. Smereka, Poverkhn., Fiz. K'him. Mekh. 8, 24 (1985).

[4] P. Soukiassian, R.S. List, J.C. Woicik, P. Pianetta, I. Lindau, W.E. Spicer, Phys. Rev. B 37, 7115 (1988).

[5] P. Soukiassian, M.H. Rakshi, H.I. Starnberg, Z. Hurych, T.M. Gentle, K.P. Schuette, Phys. Rev. Lett. 59, 1489 (1987).

[6] N. Ernst, W. Drechsel, J. Li, J.H. Block, H.J. Kreutzer, Phys. Rev. Lelt. 57, 2686 (1986).

[7] K. Nath, H.J. Kreutzer, A.B. Anderson, Surf. Sci.176, 261 (1986).

[8] S.C. Wang, T.T. Tsong, Phys. Rev. B 26, 647 (1982).

[9] E.V. Klimenko, A.G. Naumovets, Surf. Sci. 14, 141 (1969). 
[10] E.V. Klimenko, A.G. Naumovets, Fiz. Tverd. Tela 13, 33 (1971).

[11] E.V. Klimenko, A.G. Naumovets, Fiz. Tverd. Tela 15, 3273 (1973).

[12] G.G. Vladimirov, B.K. Medvedev, I.L. Sokolskaya, Fiz. Tverd. Tela 12, 539 (1970).

[13] G.G. Vladimirov, S.S. Lobanova, Fiz. Tverd. Tela 15, 3169 (1973).

[14] Yu. Suchorski, Surf. Sci. 231, 130 (1990).

[15] Yu. Suchorski, Surf. Sci. 247, 346 (1991).

[16] C.J. Todd, T.N. Rlodin, Surf. Sci. 42, 109 (1974).

[17] W.D. McNeil, W.T. Shepherd, Rev. Sci. Instrum. 43, 1636 (1972).

[18] W.G. Ivanov, G.N. Fursey, I.L. Sokolskaya, Fiz. Tverd. Tela 0, 1144 (1967).

[19] E.W. Plummer, T.N. Rlıdin, J. Chem. Phys. 49, 3479 (1968).

[20] G.L. Kellog, Phys. Rev. 28, 1957 (1983).

[21] P.G. Borzjak, A.A. Dadykin, Pis'ma Zh. Exp. Teor. Fiz. (USSR) 40, 269 (1984).

[22] V.K. Medvedev, A.I. Yakivtchuk, Structure, Electron and Adsorption Properties of Alkali Adlayers on the (111) Plane of Tungsten Single Crystal, Acad. of Science Ukr. SSR, Inst. of Pliys., Kiev 1975, p. 47.

[23] M. Drechsler, Z. Elektrochem. 61, 48 (1957).

[24] D.M. Riffe, G.K. Wertheim, P.II. Citrin, Phys. Rev. Lett. 64, 571 (1990).

[25] D.W. Bassett, M.J. Parsley, J. Phys. D 2, 13 (1969).

[26] H. Tochihara, M. Kubota, M. Miyao, Y. Murata, P. Soukiassian, Surf. Sci. 172, L507 (1986).

[27] K. Horn, A. Hohlfeld, I. Somers, T. Lindner, P. Hollins, A.M. Bradshaw, Phys. Rev. Lett. 61, 2488 (1988).

[28] A. Dalgarno, Adv. Phys. 11, 281 (1962).

[29] G.I. Bigun, Yu, Suclorski, Surf. Sci. 247, 111 (1991).

[30] G.I. Bigun, I.D. Nabitovich, Yu. Suchorski, Fiz. Tverd. Tela 23, 2128 (1981).

[31] J. Bęben, Ch. Kleint, R. Mçclewski, Appl. Phys. A 40, 79 (1986).

[32] J. Bęben, Ch. Kleint, R. Męclewski, Z. Phys. B 69, 319 (1987).

[33] Ya.B. Lozovy, Ph.D. Thesis, Lviv University, Lvov 1984, p. 32.

[34] A.I. Volokitin, Fiz. Tekh. Poluprovodn. (USSR) 8, 1648 (1979). 